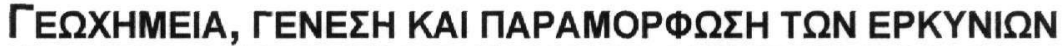

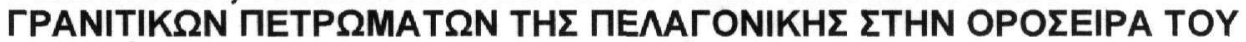

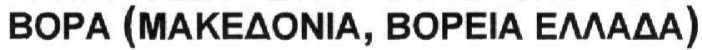

\author{
Auүepıvás $A .{ }^{1}$, Kíiıas $\Delta .{ }^{1}$, Kopwvaíos $A .{ }^{2}$ kaı Mouvtpákns $\Delta .{ }^{1}$

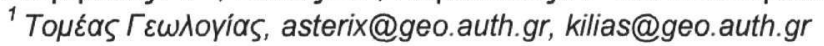

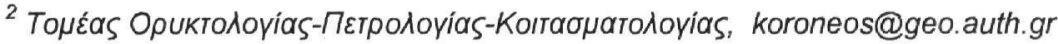

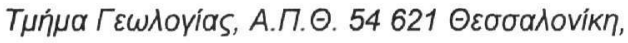

\section{ПЕРІ^НЧH}

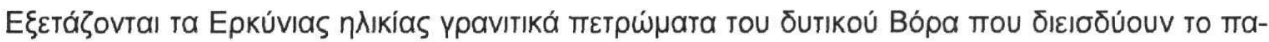

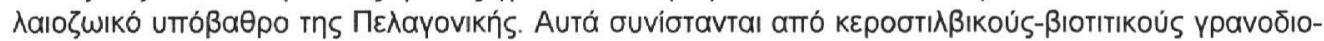

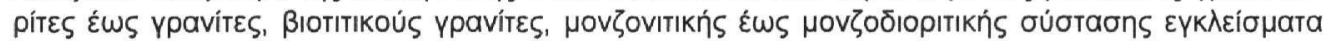

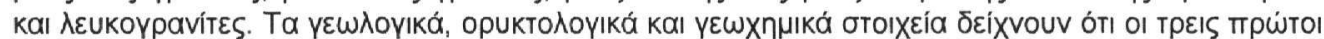

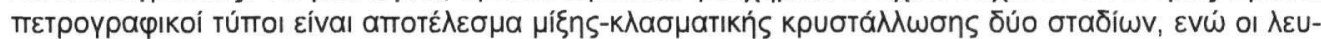

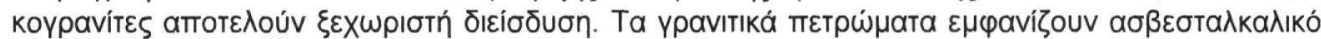

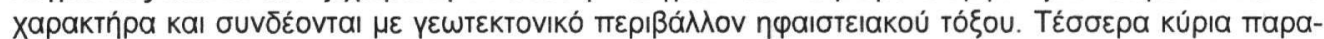

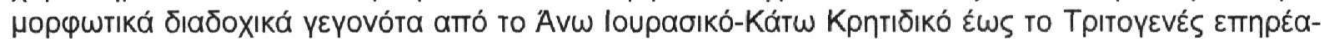

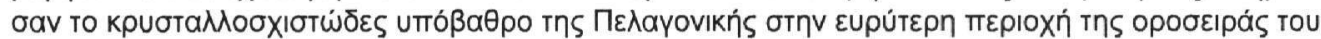

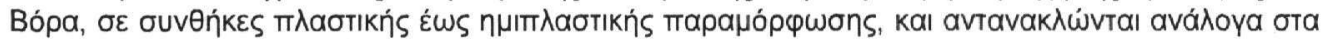

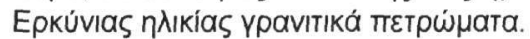

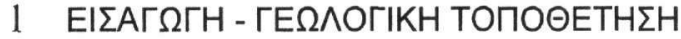

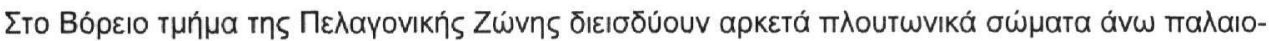

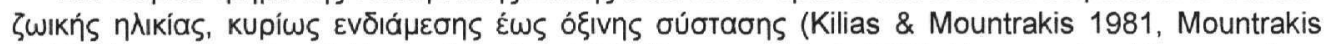

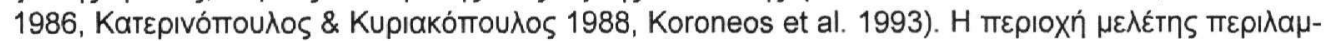

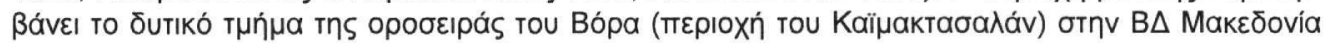

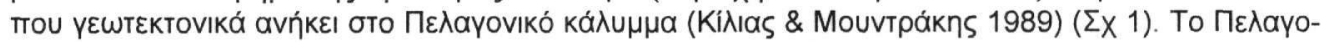

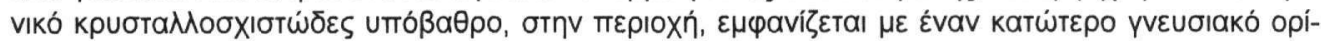

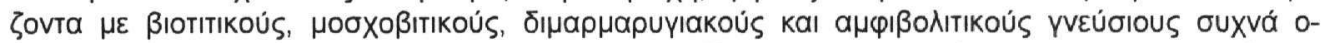

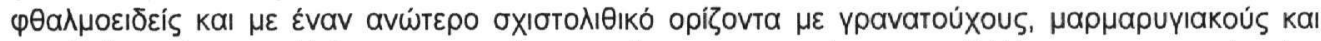

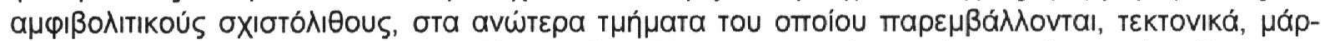

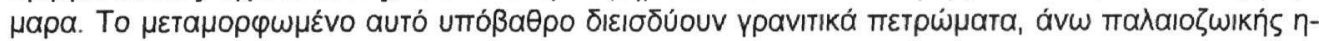

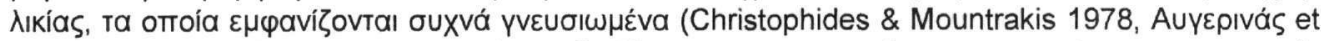

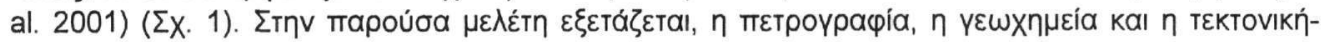

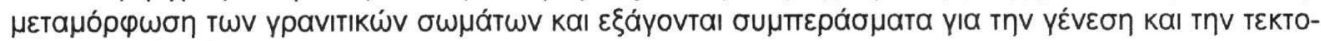
VIKń TOU $\varepsilon \xi \dot{\varepsilon} \lambda \mid \xi \eta$.

\section{2 ПЕТРОГРАФІА - ОРYКТОЛОГІА}

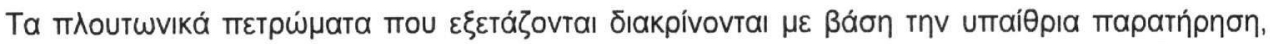

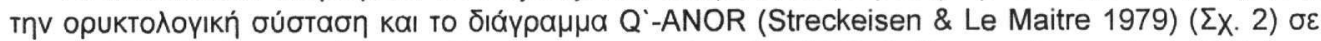

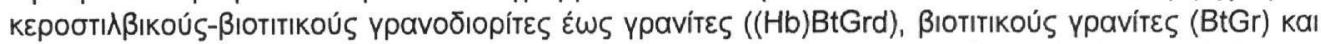

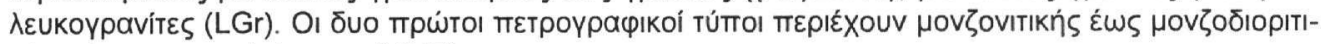

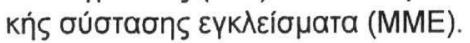

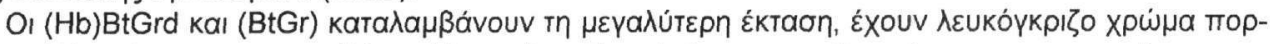

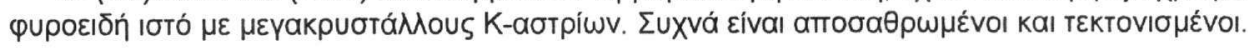




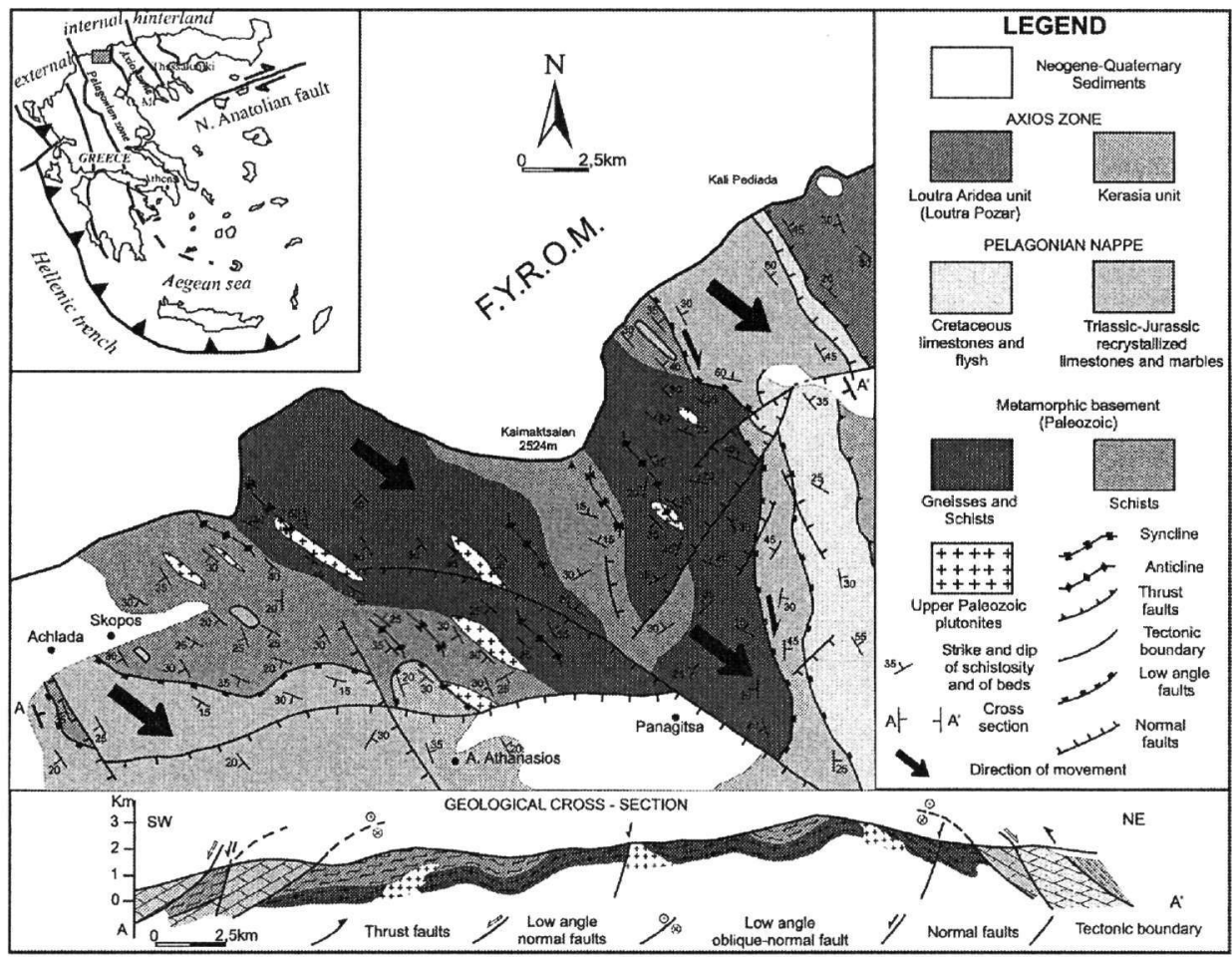

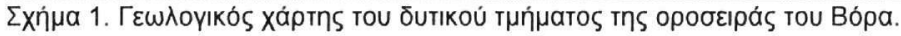

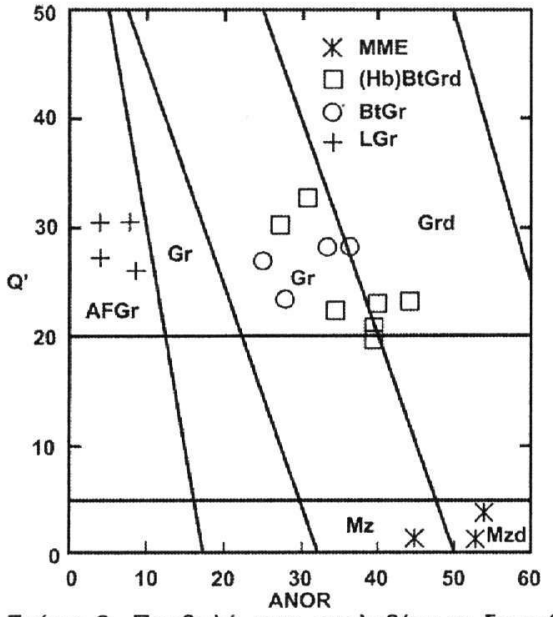

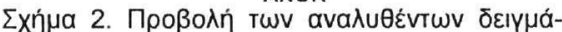

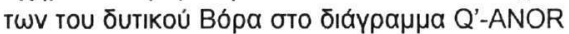
(Streckeisen \& Le Maitre 1979). AFGr: $a \lambda k \alpha \lambda l-$

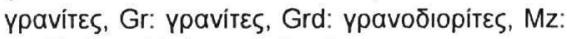

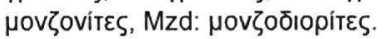

Ta трштоуєvп́ ориктá бтоus (Hb)BtGrd кaı

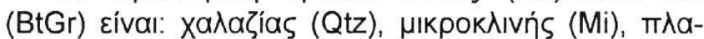

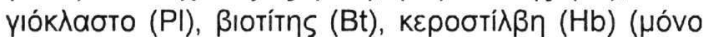

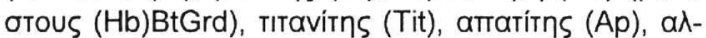

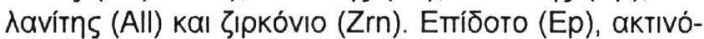

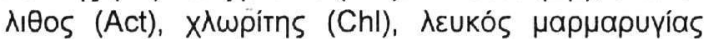

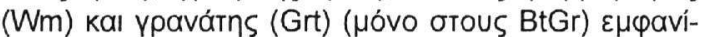

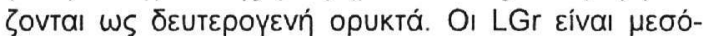

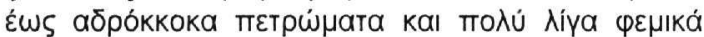

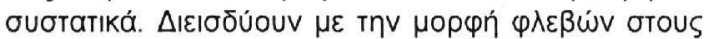

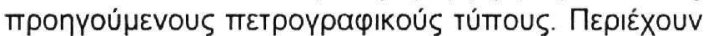

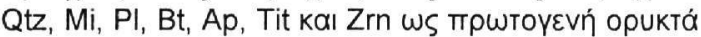

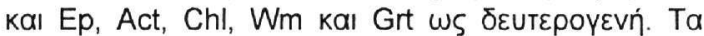

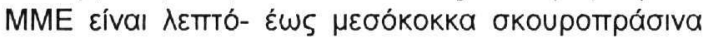

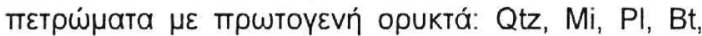

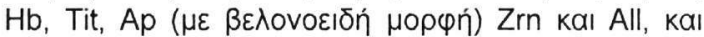

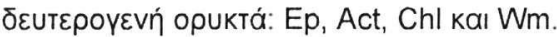

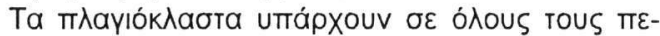

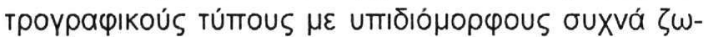

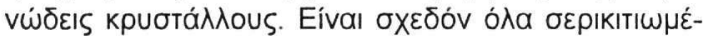

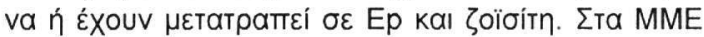

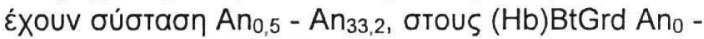

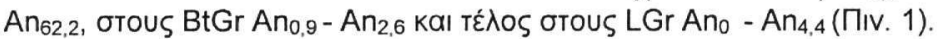

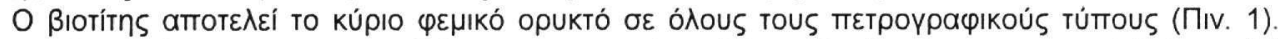

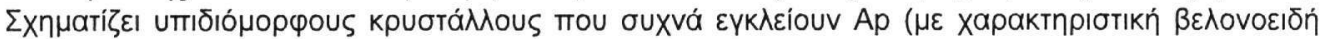

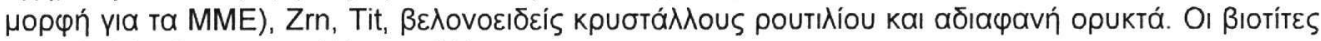

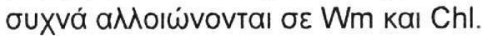




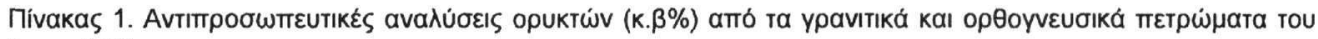
ठutıkoú Bópa.

\begin{tabular}{|c|c|c|c|c|c|c|c|c|c|c|c|c|c|c|c|c|c|}
\hline \multirow{3}{*}{ 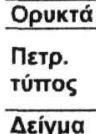 } & \multicolumn{9}{|c|}{ Bıоті́теS } & \multicolumn{5}{|c|}{ 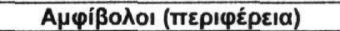 } & \multicolumn{3}{|c|}{ 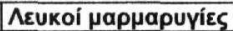 } \\
\hline & \multicolumn{4}{|c|}{ (Hb)BtGrd } & \multicolumn{2}{|c|}{ BtGrt } & \multicolumn{2}{|c|}{ MME } & \multirow{2}{*}{\begin{tabular}{|c|} 
LGr \\
6.3
\end{tabular}} & \multicolumn{4}{|c|}{ (Hb)BtGrd } & \multirow{2}{*}{\begin{tabular}{|c|} 
MME \\
$1.2 \varepsilon$
\end{tabular}} & \multicolumn{2}{|c|}{$\mathrm{BtGr}$} & \multirow{2}{*}{$\begin{array}{c}\begin{array}{c}\text { Ortho- } \\
\text { gneis- } \\
\text { ses }\end{array} \\
6.13 \\
\end{array}$} \\
\hline & 1.2 & 6.10 & 1.1 & 1.2 & 211.2 & 6.2 & 6.4 & $1.2 \varepsilon$ & & 1.2 & 6.10 & 6.6 & 1.1 & & 211.2 & 211.1 & \\
\hline $\mathrm{SiO}_{2}$ & 36,52 & 36,63 & 36,32 & 35,10 & 35,78 & 36,48 & 37,15 & 36,83 & 41,85 & 47,46 & 45,75 & 47,07 & 48,80 & 45,84 & 46,65 & 48,84 & 49,41 \\
\hline $\mathrm{TiO}_{2}$ & 2,16 & 1,88 & 2,76 & 2,79 & 32 & 1,76 & 1,77 & 2,36 & 1,60 & 0,62 & 0,97 & 0,97 & 0,66 & 1,17 & 1,20 & 0,55 & 0,52 \\
\hline $\mathrm{Al}_{2} \mathrm{O}_{3}$ & 15,59 & 15,64 & 14,71 & 15,99 & 15,11 & 15,29 & 16,28 & 16,09 & 17,91 & 5,95 & 7,14 & 6,50 & 3,86 & 7,22 & 28,62 & 28,01 & 26,20 \\
\hline eOt & 19,51 & 19,05 & 20,62 & 22,26 & 21,41 & 21,83 & 17,91 & 17,30 & 14,70 & 17,25 & 16,45 & 17,03 & 15,12 & 16,53 & 4,33 & 4,59 & 4,17 \\
\hline InO & 0,28 & 0,30 & 0,36 & 0,22 & 46 & 0,21 & 0,46 & 0,14 & 0,57 & 0,73 & 63 & 54 & 65 & 0,45 & 0,09 & & 0,19 \\
\hline $\lg \mathrm{O}$ & 11,61 & 11,82 & 11,05 & & & 8 , - & 12,15 & 12,24 & 10,10 & 11,75 & 12,03 & 11,98 & 14,03 & 12,01 & 2,29 & 2,70 & 3,17 \\
\hline & 0,00 & 0,01 & 0,00 & 0,00 & 0,00 & 0,01 & 0,00 & 0,01 & 0,04 & 12,15 & 12,33 & 12,36 & 12,50 & 11,98 & & & \\
\hline $\mathrm{a}_{2} \mathrm{O}$ & 0,23 & 0,36 & 0,26 & 0,22 & 0,34 & 0,33 & 0,28 & 0,33 & 0,20 & 1,35 & 1,64 & 1,51 & 0,67 & 1,56 & 0,27 & 0,27 & 0,27 \\
\hline 0 & 9,44 & 9,20 & 9,59 & 9,33 & 9,04 & 9,05 & 9,71 & 9,30 & 8,05 & 0,59 & 0,64 & 0,64 & 0,48 & 0,79 & 10,55 & 10,95 & 10,05 \\
\hline$J_{3}$ & & & & & & & & & & 0,11 & 0,14 & 0,04 & 0,12 & 0,00 & & & \\
\hline Total & 95,34 & 94,90 & 95,65 & 94,92 & 94,32 & 93,83 & 95,71 & 94,58 & 95,01 & 97,94 & 97,72 & 98,60 & 96.89 & 97,54 & 93,94 & 95,91 & 93,98 \\
\hline
\end{tabular}

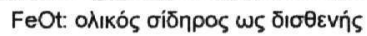
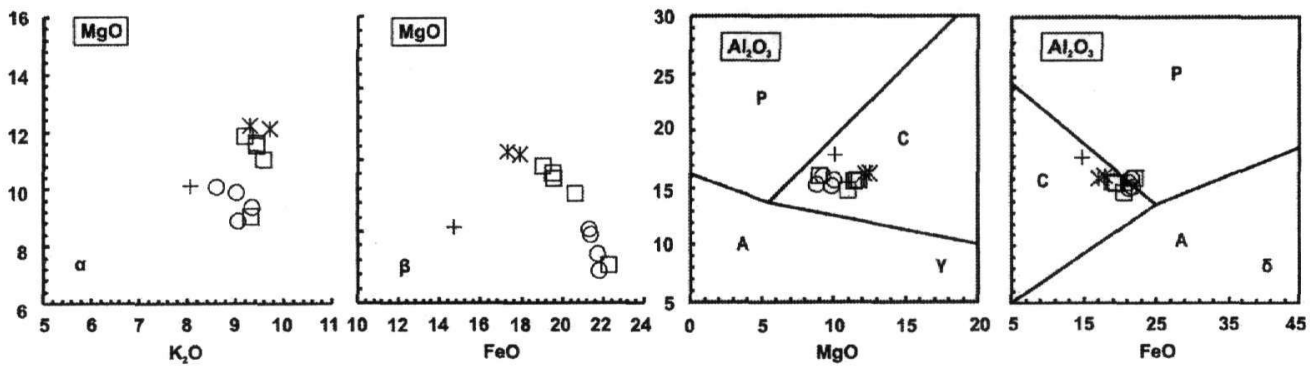

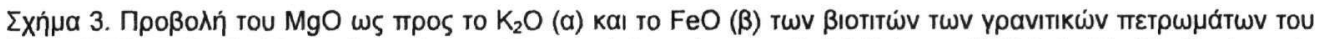

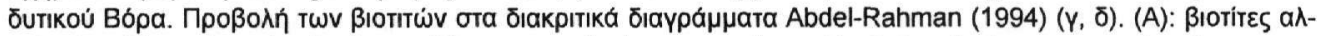

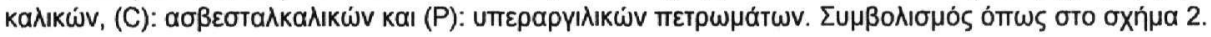

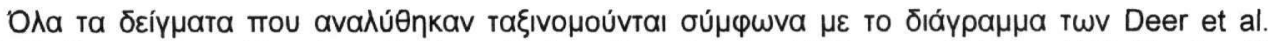

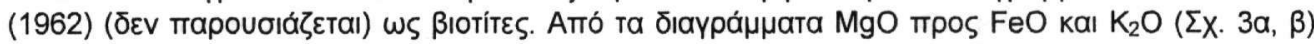

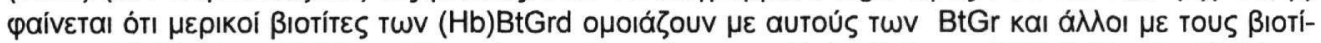

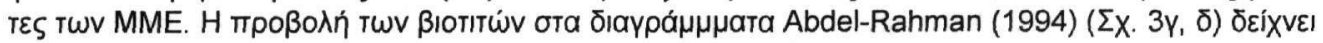

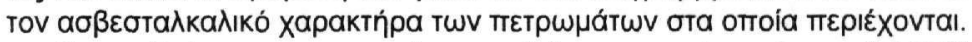

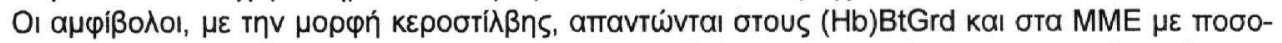

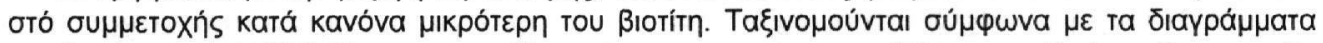

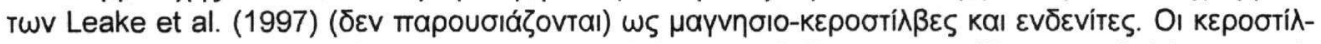

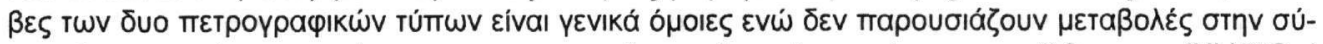

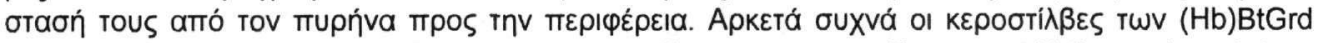

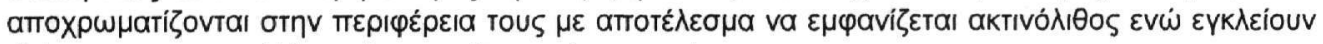

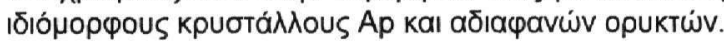

\section{3 ГE $\Omega X H M E I A$}

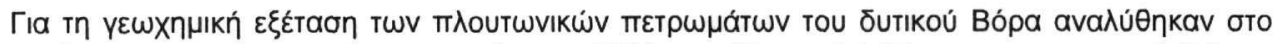

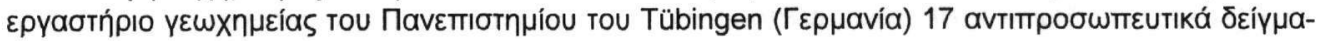

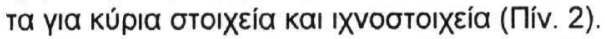

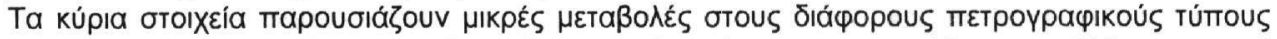

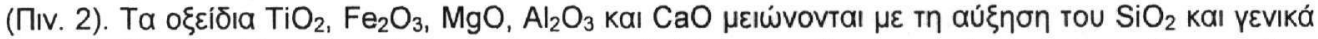

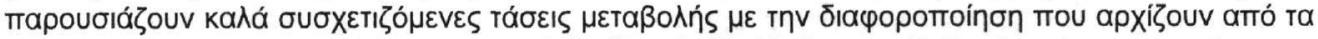

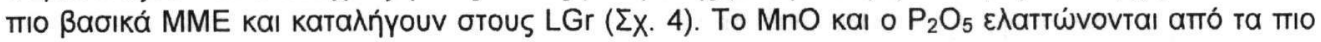

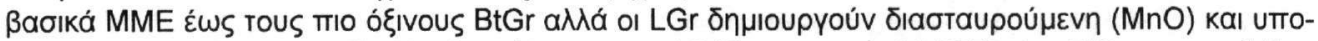

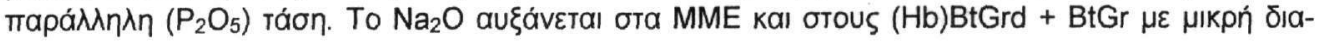




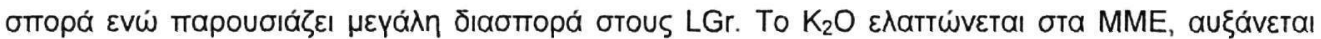

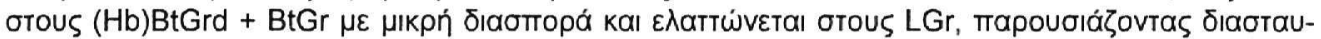

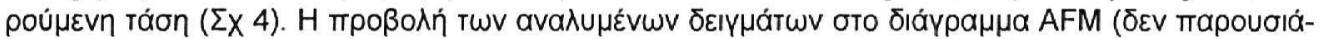

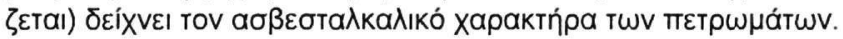

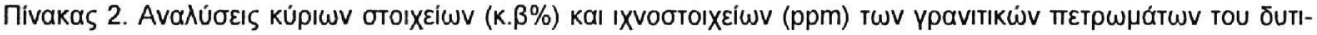
koú Bópa.

\begin{tabular}{|c|c|c|c|c|c|c|c|c|c|c|c|c|c|c|c|c|c|}
\hline \multirow{2}{*}{ 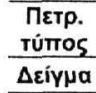 } & \multicolumn{3}{|c|}{ MME } & \multicolumn{6}{|c|}{ (Hb)BtGrd } & \multicolumn{4}{|c|}{$\mathrm{BtGr}$} & \multicolumn{4}{|c|}{ LGr } \\
\hline & 6.4 & $1.1 \varepsilon$ & $1.2 \varepsilon$ & 1.2 & 6.9 & 6.10 & 6.6 & 1.1 & 6.11 & 211.2 & 211.1 & 6.5 & 6.2 & 6.3 & 6.7 & 1.3 & 6.12 \\
\hline $\mathrm{SiO}_{2}$ & 47,13 & 50,13 & 54,36 & 64,76 & 65,06 & 65,07 & 65,66 & 66,72 & 66,91 & 66,40 & 67,74 & 68,65 & 71,55 & 71,60 & 73,58 & 74,47 & 75,07 \\
\hline $\mathrm{TiO}_{2}$ & 1,64 & 1.36 & 1,24 & 0,51 & 0.51 & 0,52 & 0,51 & 0,47 & 0,52 & 0,55 & 0,49 & 0,30 & 0,16 & 0,11 & 0,02 & 0,05 & 0,08 \\
\hline $\mathrm{Al}_{2} \mathrm{O}_{3}$ & 18,74 & 19,51 & 17,74 & 16,31 & 16,35 & 16,09 & 16,06 & 16,41 & 16,28 & 15,65 & 15,51 & 15,86 & 15,05 & 14,53 & 14,69 & 14,59 & 14,11 \\
\hline $\mathrm{Fe}_{2} \mathrm{O}_{3} \mathrm{t}$ & 9,84 & 8,97 & 7,77 & 3,69 & 3,76 & 3,81 & 3,69 & 3,54 & 3,72 & 3,58 & 3,20 & 2,10 & 1,16 & 0,81 & 0,35 & 0,55 & 0.62 \\
\hline $\mathrm{MnO}$ & 0.21 & 0,21 & 0,18 & 0,08 & 0,08 & 0,08 & 0,07 & 0,08 & 0,06 & 0,09 & 0,07 & 0,05 & 0,04 & 0,01 & 0,03 & 0,03 & 0,04 \\
\hline $\mathrm{MgO}$ & 5.13 & 4,65 & 4,69 & 1,76 & 1,96 & 1,80 & 1,75 & 1,74 & 1,58 & 1,23 & 1,10 & 0,80 & 0,37 & 0,28 & 0,08 & 0,09 & 0.22 \\
\hline $\mathrm{CaO}$ & 5,27 & 5,01 & 4,21 & 3,17 & 2,50 & 3,16 & 3,24 & 3,05 & 2,01 & 2,61 & 2,81 & 2,18 & 1,71 & 1,19 & 0,50 & 0,40 & 0,61 \\
\hline $\mathrm{Na}_{2} \mathrm{O}$ & 3.86 & 4,65 & 4,12 & 3,81 & 3,92 & 3,64 & 3,79 & 3,81 & 3,11 & 3,16 & 3,26 & 3,96 & 4,16 & 2,63 & 4,70 & 4,44 & 4,52 \\
\hline $\mathrm{K}_{2} \mathrm{O}$ & 4,44 & 3,53 & 3,71 & 3,72 & 3,54 & 3,83 & 3,22 & 3,60 & 3,89 & 3,82 & 3,78 & 4,28 & 4,03 & 7,16 & 4,50 & 4,23 & 4,19 \\
\hline $\mathrm{P}_{2} \mathrm{O}_{5}$ & 0,72 & 0,65 & 0,52 & 0,19 & 0,20 & 0,20 & 0,20 & 0,19 & 0,21 & 0,20 & 0,20 & 0,12 & 0,10 & 0,29 & 0,12 & 0,14 & 0.13 \\
\hline LOI & 1,82 & 1,18 & 1,44 & 0,97 & 1,54 & 1,03 & 1,06 & 0,78 & 1,61 & 1,60 & 1,44 & 0,95 & 0,68 & 0,57 & 0,46 & 0,59 & 0,48 \\
\hline $\mathrm{Rb}$ & 269 & 225 & 205 & 142 & 137 & 144 & 135 & 138 & 171 & 202 & 176 & 184 & 182 & 198 & 219 & 428 & 162 \\
\hline $\mathrm{Sr}$ & 536 & 520 & 494 & 624 & 549 & 576 & 586 & 622 & 450 & 268 & 268 & 335 & 247 & 350 & 35 & 36 & 130 \\
\hline $\mathrm{Ba}$ & 772 & 1158 & 740 & 1276 & 1109 & 1290 & 896 & 1158 & 1087 & 646 & 616 & 772 & 399 & 1625 & 46 & 94 & 448 \\
\hline $\mathrm{Zn}$ & 120 & 111 & 110 & 48 & 53 & 52 & 52 & 45 & 56 & 82 & 51 & 38 & 20 & 10 & 6 & 27 & 7 \\
\hline $\mathrm{Ni}$ & 5 & 26 & 42 & 22 & 11 & 23 & 29 & 23 & 16 & 3 & * & 10 & 2 & 2 & 1 & 2 & * \\
\hline $\mathrm{Cr}$ & 40 & 113 & 154 & 59 & 96 & 48 & 51 & 38 & 49 & 7 & 5 & 40 & 18 & 2 & 3 & 5 & * \\
\hline V & 256 & 190 & 163 & 62 & 65 & 62 & 58 & 56 & 65 & 56 & 48 & 26 & 17 & 6 & 5 & 5 & 6 \\
\hline$Y$ & 52 & 48 & 34 & 20 & 18 & 16 & 18 & 19 & 20 & 26 & 20 & 18 & 12 & 22 & 10 & 13 & 11 \\
\hline $\mathrm{Zr}$ & 334 & 308 & 305 & 194 & 200 & 196 & 202 & 195 & 196 & 165 & 153 & 138 & 86 & 56 & 32 & 35 & 40 \\
\hline $\mathrm{Nb}$ & 22 & 22 & 18 & 10 & 8 & 8 & 7 & 8 & 10 & 14 & 12 & 7 & 9 & * & 5 & 19 & 18 \\
\hline
\end{tabular}

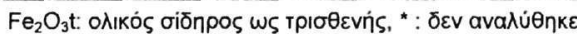

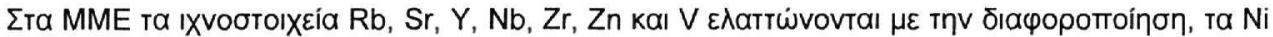

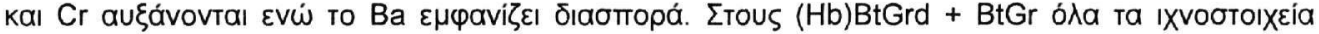

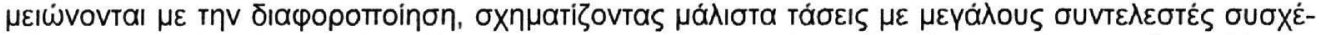

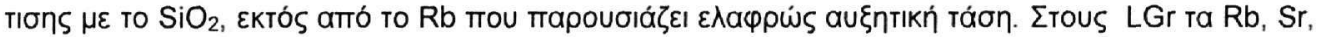

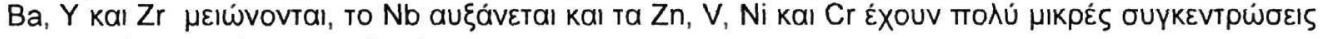

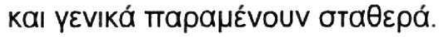

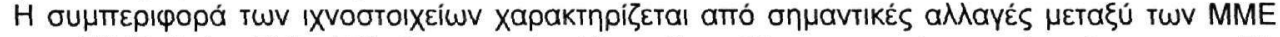

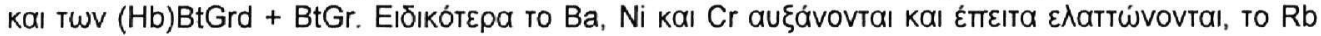

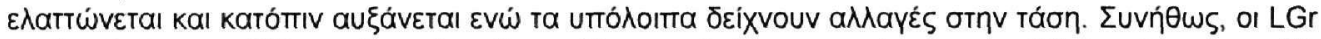

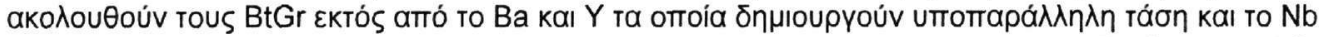

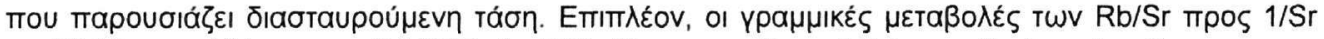

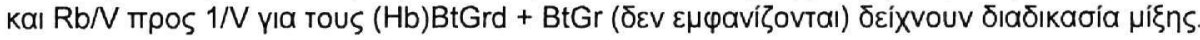

\section{4 ГESTEKTONIKO ПEPIBAM $\wedge O N$}

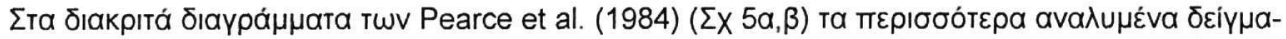

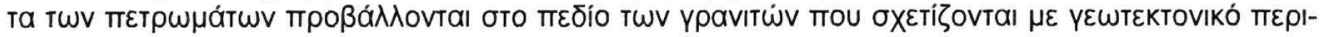

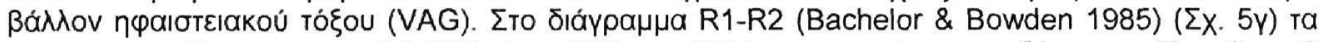

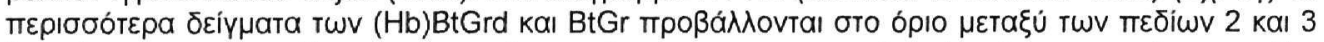

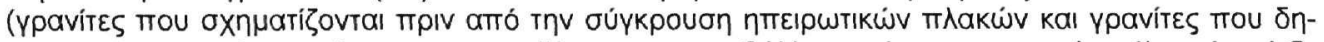

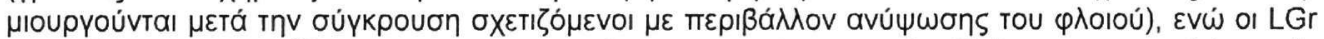

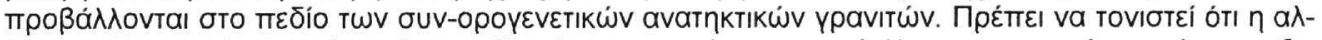

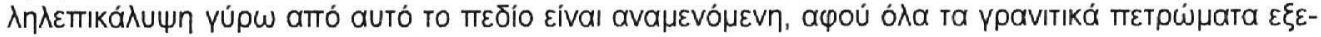

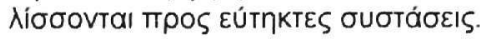



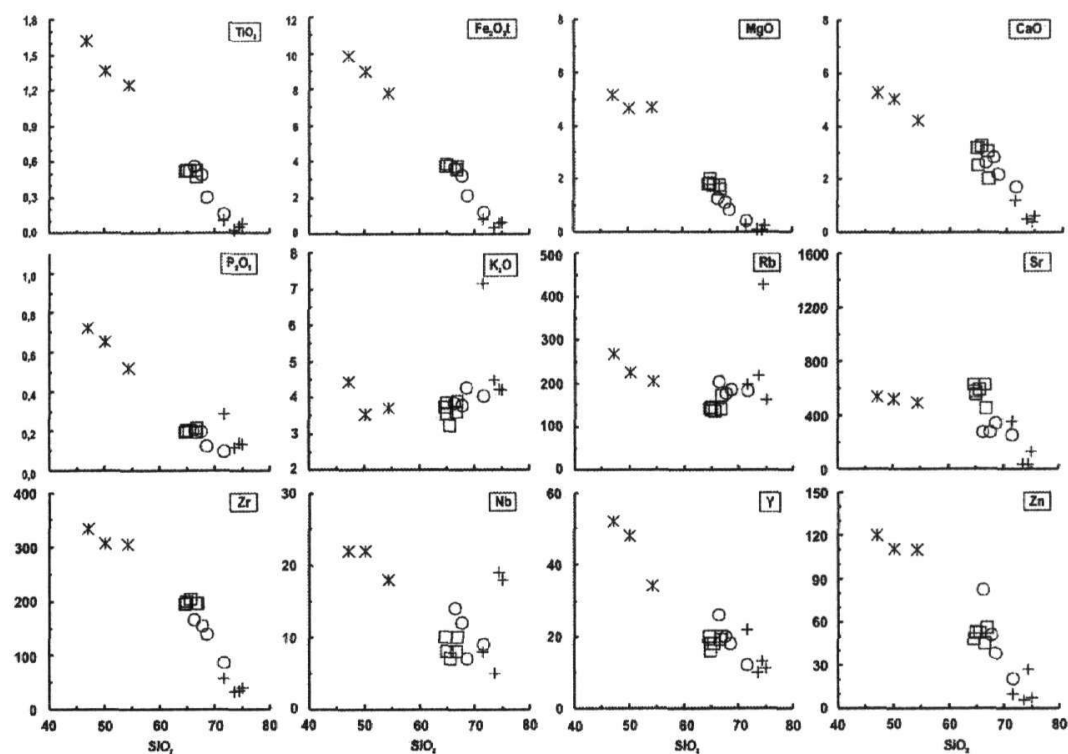

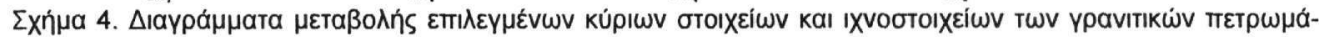

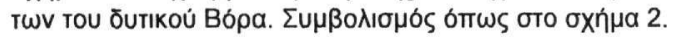
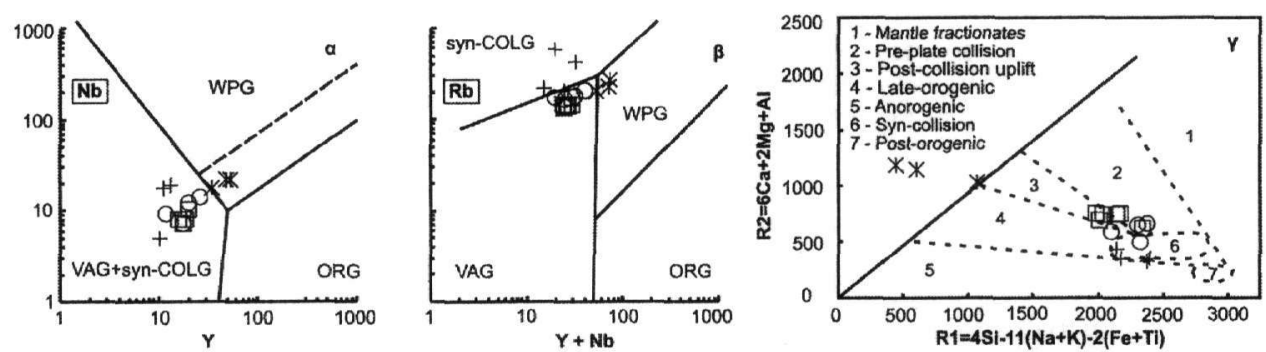

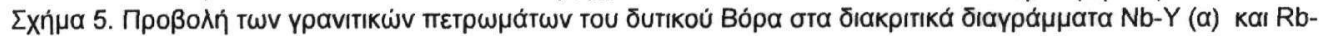

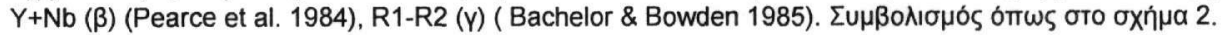

\section{TEKTONIKH - METAMOPФ $\Omega \Sigma H$}

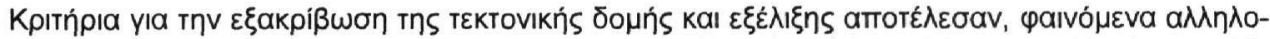

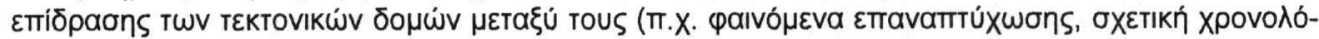

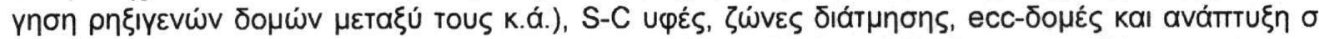

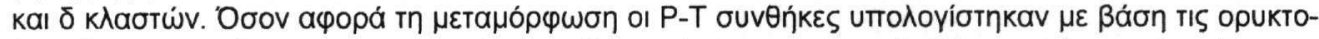

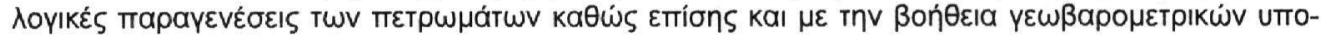

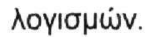

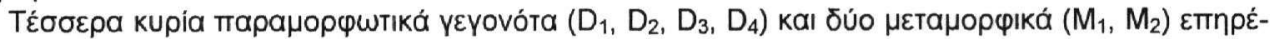

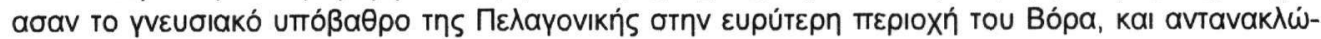

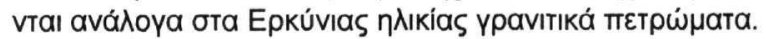

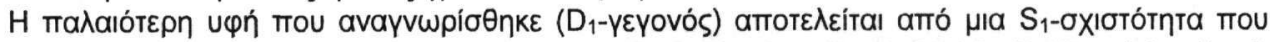

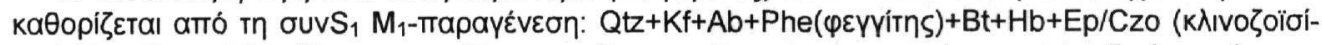

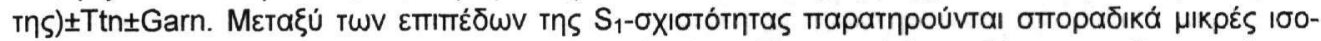

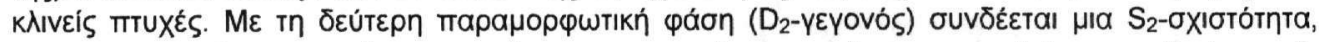

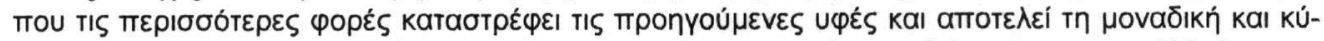

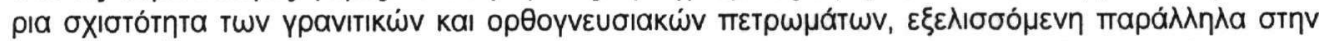

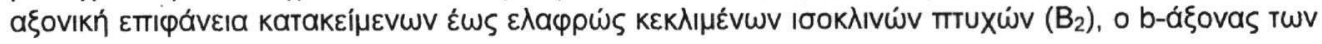




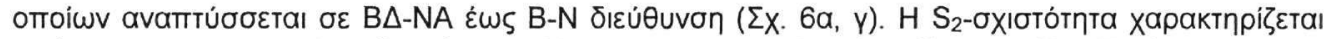

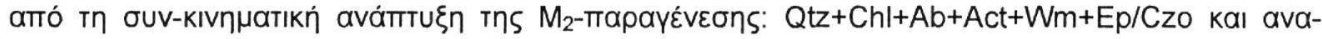

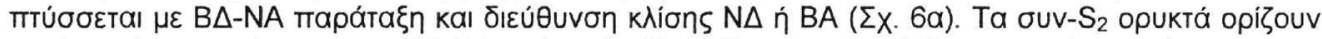

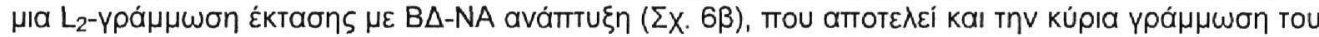

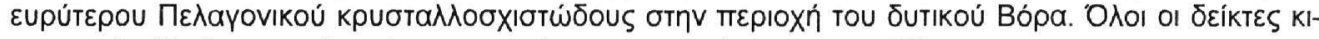

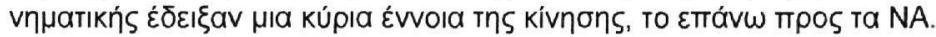

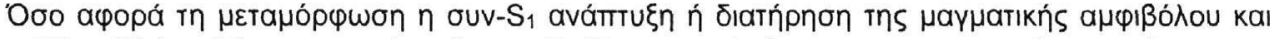

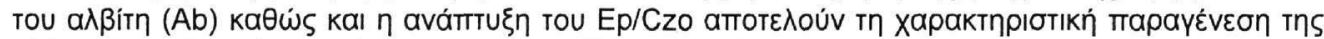

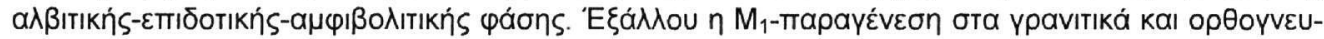

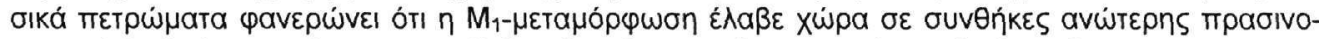

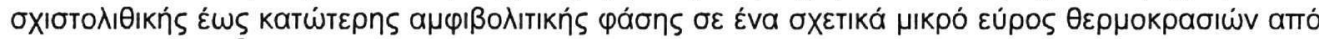

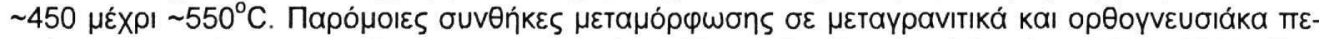

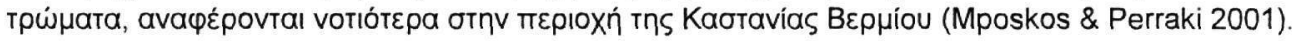

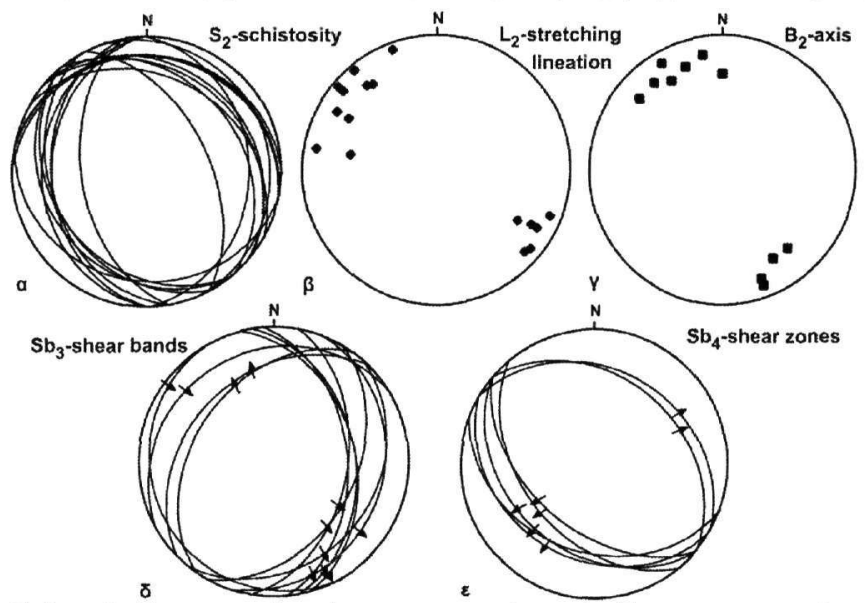

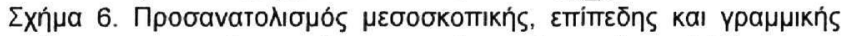

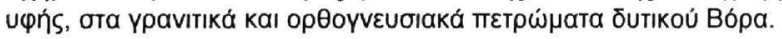

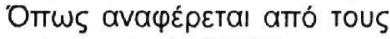

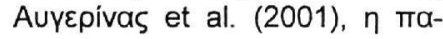

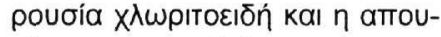

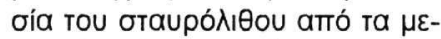

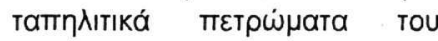

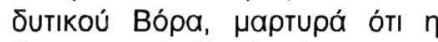

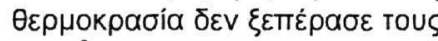

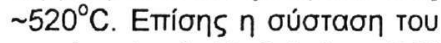

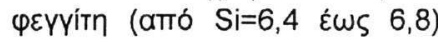

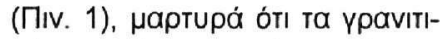

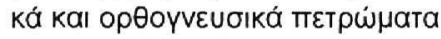

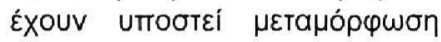

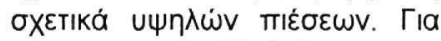

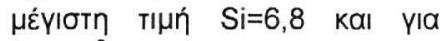

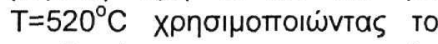

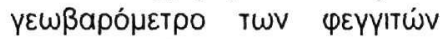
(Massone \& Schreyer 1987) $\eta$

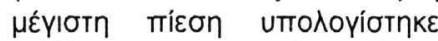

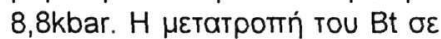

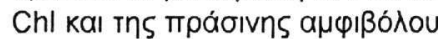

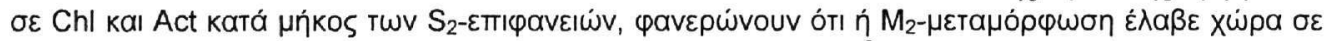

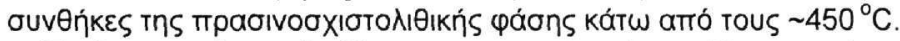

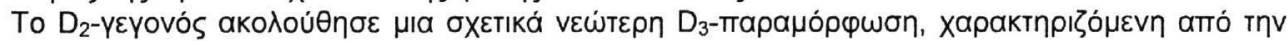

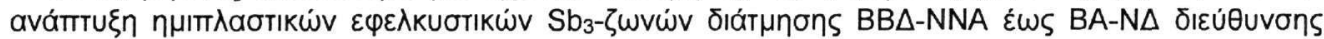

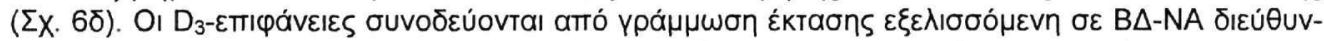

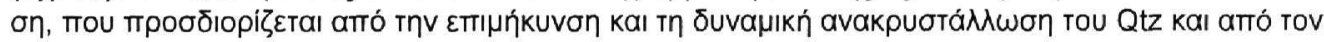

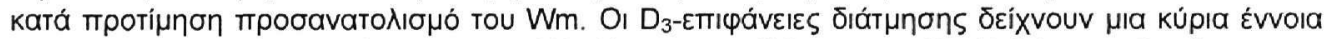

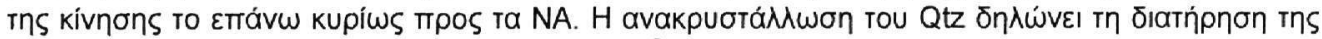

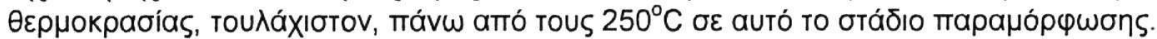

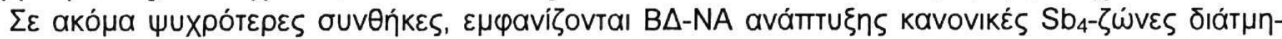

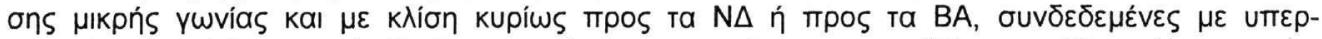

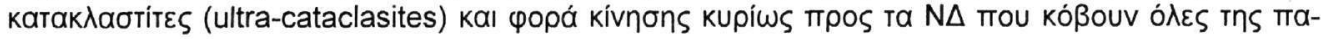

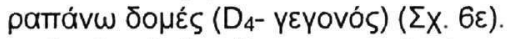

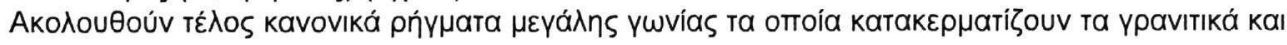

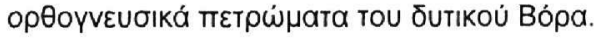

\section{$6 \quad \Sigma Y Z H T H \Sigma H-\Sigma Y M \Pi E P A \Sigma M A T A$}

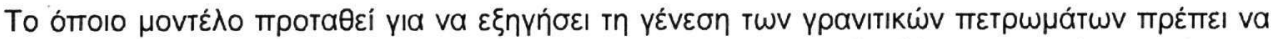

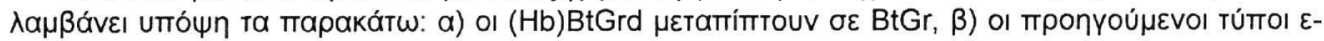

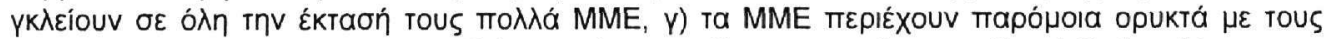

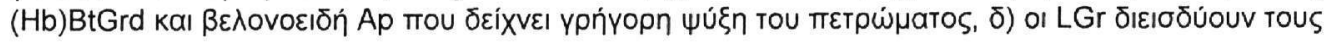




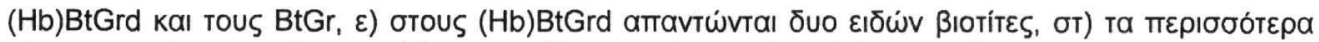

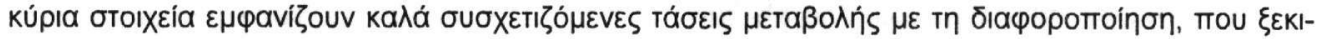

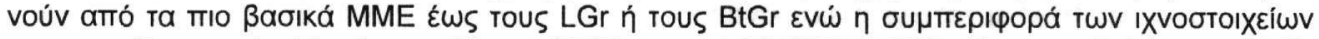

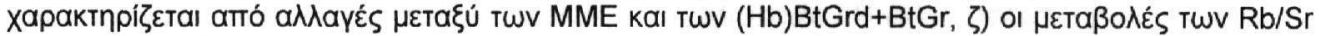

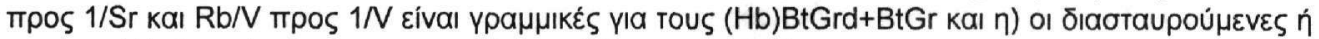

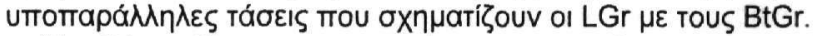

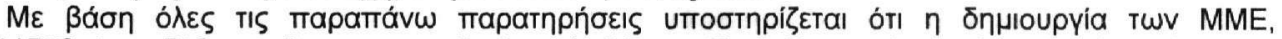

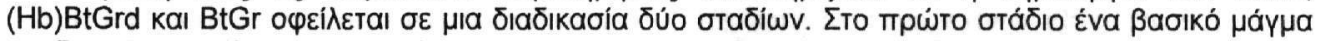

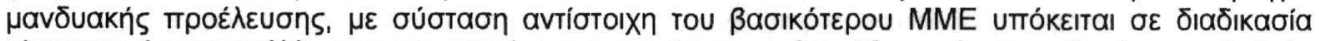

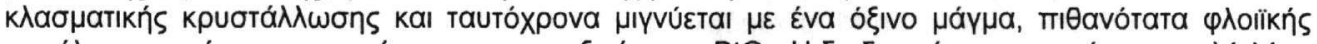

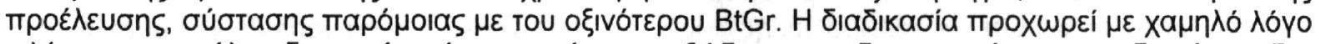

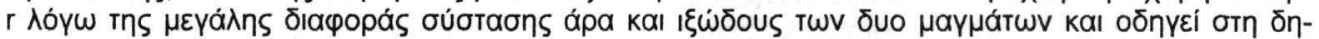

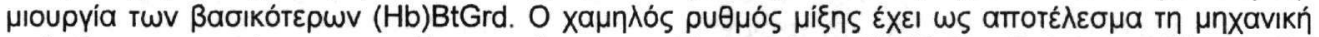

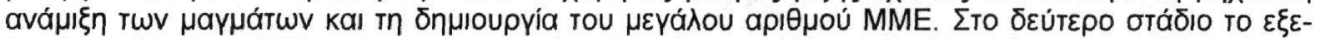

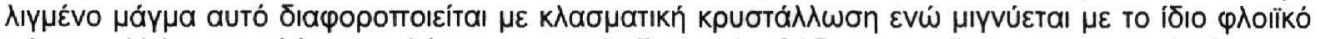

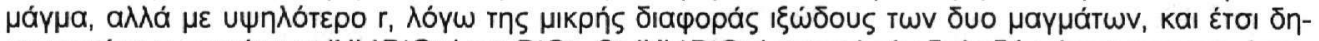

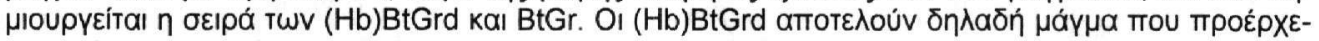

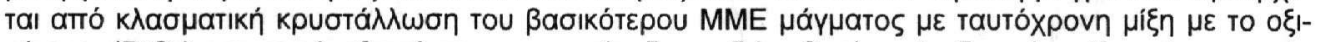

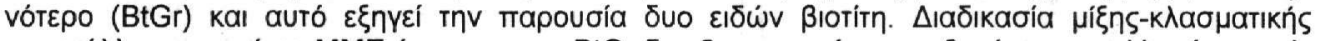

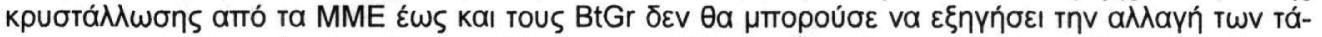

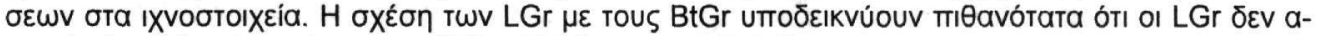

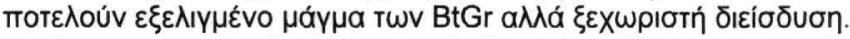

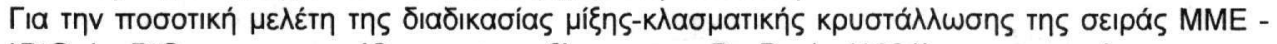

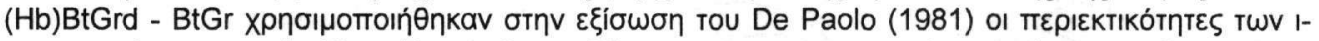

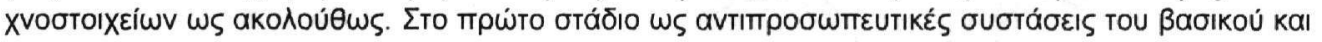

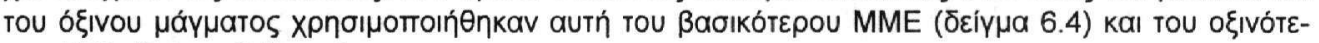

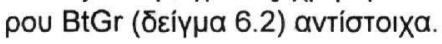

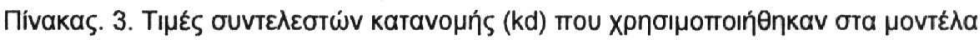

\begin{tabular}{l|cccccccc}
\hline OpukTá & $\mathbf{N b}$ & $\mathbf{Z r}$ & $\mathbf{Y}$ & $\mathbf{S r}$ & $\mathbf{R b}$ & $\mathbf{Z n}$ & $\mathbf{V}$ & $\mathbf{B a}$ \\
\hline PI & $0,060^{1}$ & $0,100^{1}$ & $0,030^{4}$ & $2,600^{4}$ & $0,760^{7}$ & $0,240^{2}$ & $0,130^{5}$ & $0,110^{4}$ \\
Kf & $0,150^{2}$ & $0,030^{2}$ & $0,086^{2}$ & $1,480^{2}$ & $2,400^{8}$ & & $1,100^{2}$ & $4,000^{11}$ \\
Bt & $4,600^{2}$ & $1,197^{1}$ & $2,300^{2}$ & $0,050^{3}$ & $5,300^{2}$ & $11,400^{2}$ & $2,000^{3}$ & $3,700^{13}$ \\
Hb & $6,700^{2}$ & $4,000^{1}$ & $2,400^{3}$ & $0,022^{6}$ & $0,400^{9}$ & $2,300^{9}$ & $10,000^{3}$ & $0,044^{1}$ \\
Zrn & $1,000^{3}$ & $2000^{3}$ & $71,400^{5}$ & & & & & \\
Ap & $0,100^{1}$ & $0,100^{1}$ & $40,000^{1}$ & & $0,400^{11}$ & & & \\
Mt & $2,500^{1}$ & $0,800^{1}$ & $0,120^{2}$ & & & $11,900^{2}$ & $8,700^{12}$ & \\
Tit & $6,300^{1}$ & & & & & $3,900^{10}$ & & \\
Qtz & & & & & $0,041^{1}$ & & & $0,022^{1}$ \\
\hline
\end{tabular}

1: Rollinson (1993), 2: Ewart \& Griffin (1994), 3: Soldatos et al. (1998), 4: Ewart et al. (1973), 5: Bea et al. (1994), 6: Nagasawa \& Schnetzler (1971), 7: Icenhower \& London (1996), 8: Nash \& Crecraft (1985), 9: Bacon \& Druitt (1988), 10: Luhr et al. (1984),

11: Mahood \& Stimac (1990), 12: Luhr \& Carmichael (1980), 13: Mahood \& Hildreth (1983).

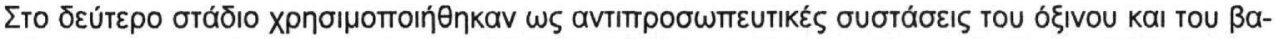

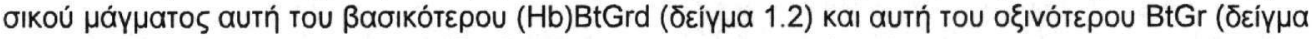

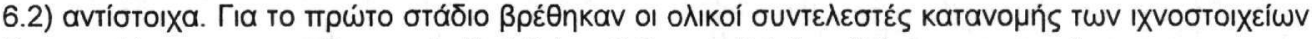

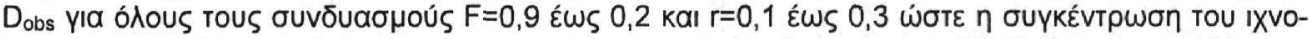

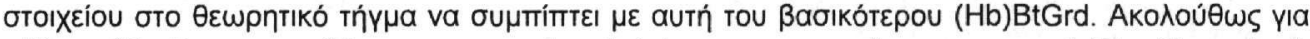

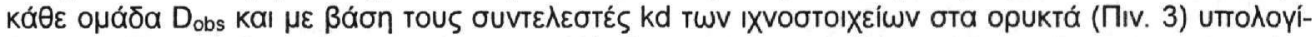

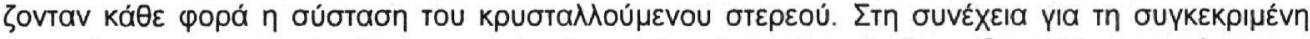

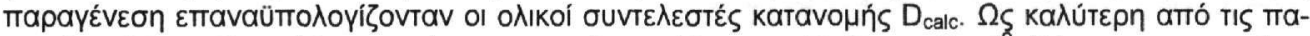

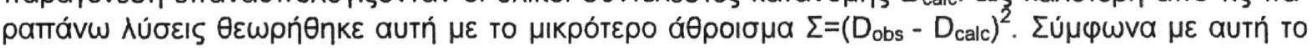

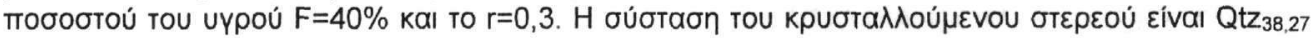

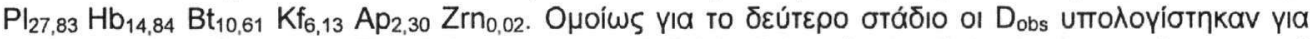

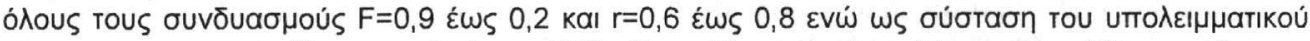

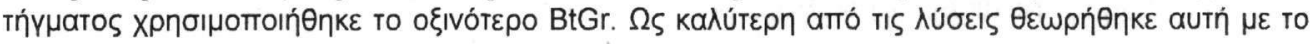




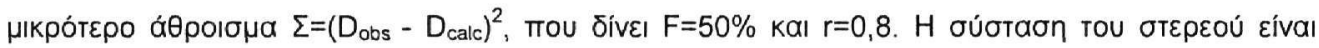
$\mathrm{Qtz}_{30,07} \mathrm{Pl}_{27,75} \mathrm{Hb}_{5,59} \mathrm{Bt}_{3,33} \mathrm{Kf}_{23,26} \mathrm{Ap}_{1,91} \mathrm{Zrn}_{0,04} \mathrm{Mt}_{2,19} \mathrm{Tit}_{5,86}$.

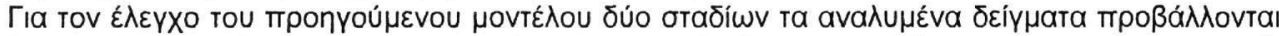

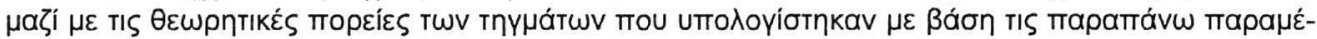

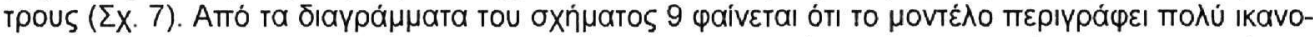
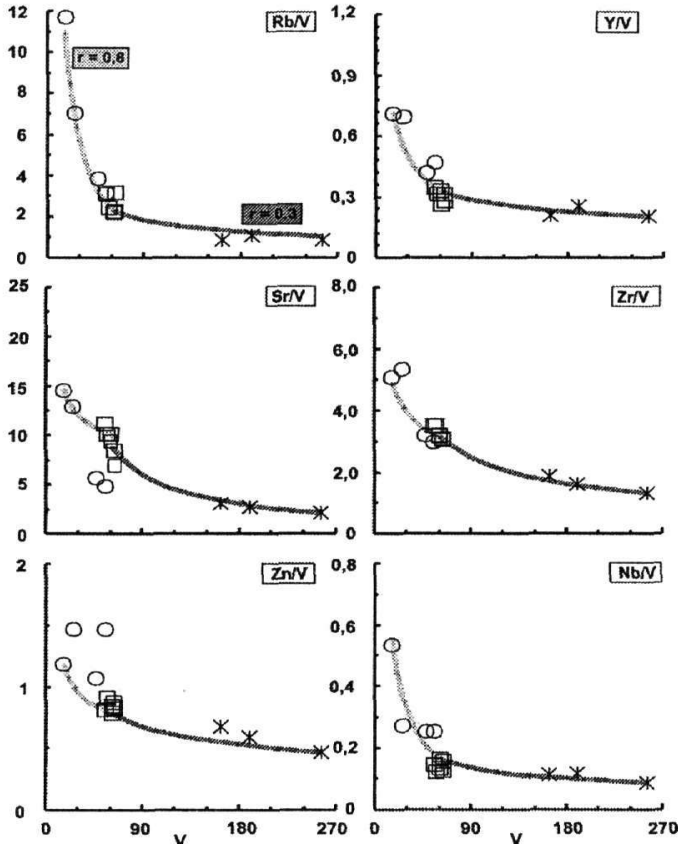

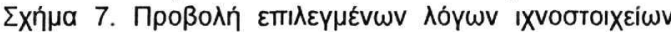

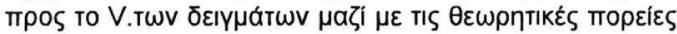

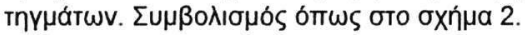

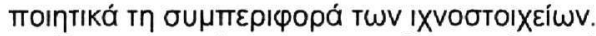

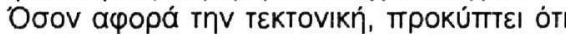

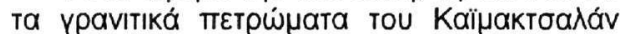

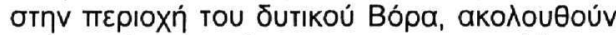

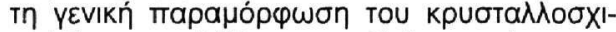

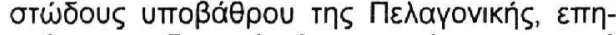

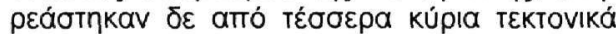

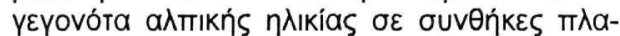

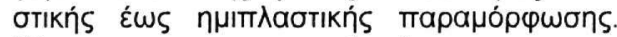
$\Sigma u ́ \mu \varphi \omega v \alpha \mu \varepsilon \quad \gamma \varepsilon \omega x p o v o \lambda$

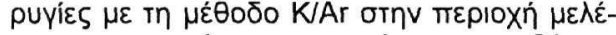

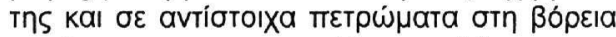

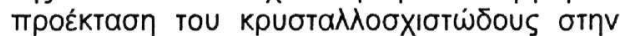
F.Y.R.O.M (Auyepivás et al. 2001, Most et al.

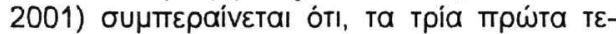

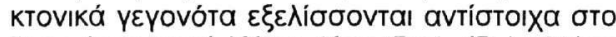

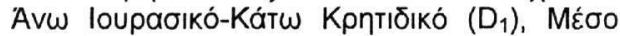

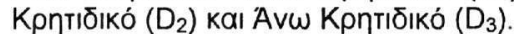

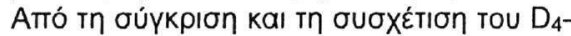

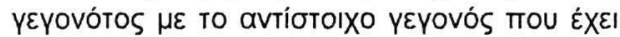

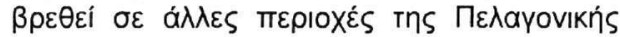

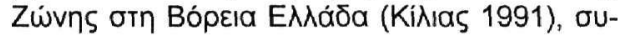

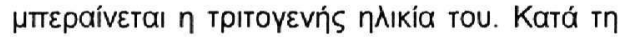

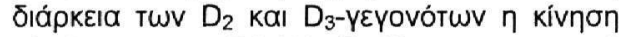

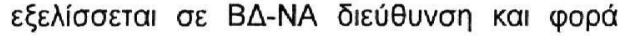

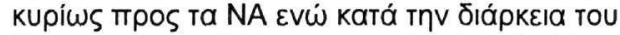

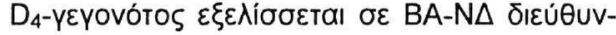

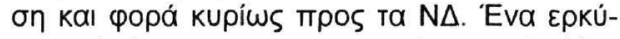

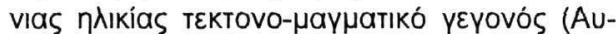

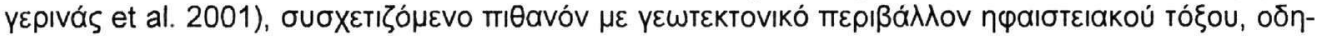

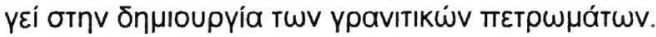

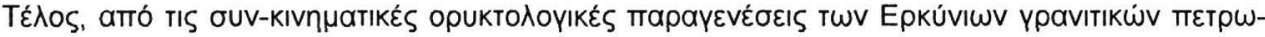

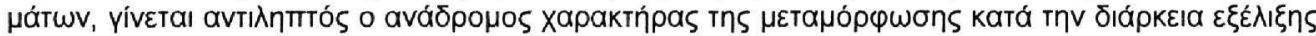

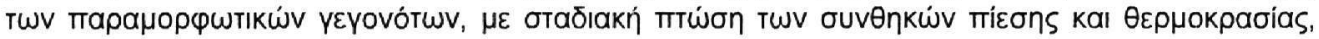

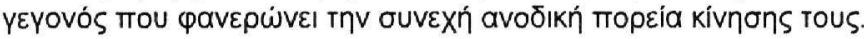

\section{ANAФOPE $\Sigma$}

Abdel - Rahman A-F. M. 1994. Nature of Biotites from Alkaline, Calk-alkaline and Peraluminous Magmas. J. Petrol., 35, 2, 525-1029.

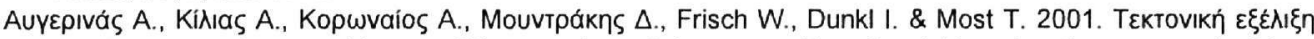

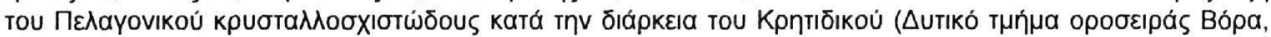

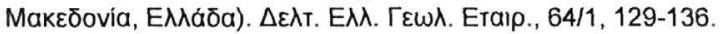

Bacon, C. R. \& Druitt, T. H. 1988: Compositional evolution of the zoned calcalkaline magma champer of Mount Mazama, Crater Lake, Oregon. Contrib. Mineral. Petrol., 98, 224-256.

Bea, F., Pereira, M. D. \& Stroh 1994. Mineral/leucosome trace-element partitioning in a peraluminous migmatite (a laser ablation-ICP-MS study). Chem. Geol., 117, 291-312.

Batchelor R. A. \& Bowden P. 1985. Petrogenetic interpretation of granitoid rocks series using multicationic parameters. Chem. Geol., 48, 43-55.

Christiphides G. \& Mountrakis D. 1978. About an occurrence of granodiorite in the North Pelagonian zone. Sci. Ann. Fac. Phys. Mathem. Univ. Thessaloniki, 18, 57-72.

De Paolo, D.J. 1981. Trace element and isotopic effects of combined wall-rock assimilation and fractional crystallization. Earth Planet. Sci. Letters, 53, 189-202. 
Deer W. A., Howie R. A. \& Zussman J. 1962. Rock-forming minerals. Vol 1,2,3,4,5. Longman, London.

Ewart A. \& Griffin W. L. 1994. Application of proton-microprobe data to trace element partitioning in volcanic rocks. Chem. Geol., 117, 251-284.

Ewart A., Bryan W. B. \& Gill J. B. 1973. Mineralogy and Geochemistry of the Younger Volcanic Islands of Tonga, S. W. Pacific. J. Petrol., 14, 3, 429-465.

Icenhower J. \& London D. 1996. Experimental partitioning of $\mathrm{Rb}, \mathrm{Cs}, \mathrm{Sr}$, and $\mathrm{Ba}$ between alkali feldspar and peraluminous melt. Am. Mineral., 81, 719-734.

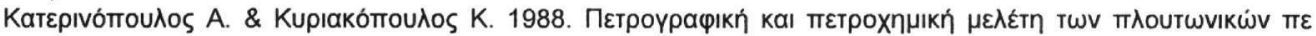

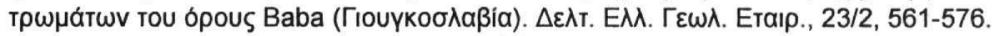

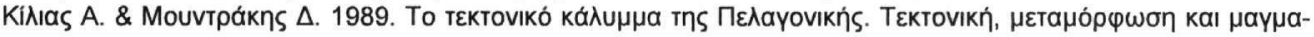

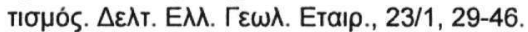

Kilias A. \& Mountrakis D. 1981. Das profil von Kaimaktsalan bis kleinen Prespa see. Die kristallinen systeme des Varnoys und des W. Kaimaktsalan (NW. Makedonien). (Beitrag zur Geologie der inneren Helleninden. Sci. Annals, Fac. Phys. 7 Mathem., Univ. Thessaliniki, 21, 95.

Kilias A. 1991. Transpressive Tektonik in den zentralen Helleniden Änderung der Translationspfade durch die Transpressione (Nord-Zentral-Griechenland). N. Jb. Geol. Paläont. Mh., 5, 291-306

Koroneos A., Christofides G., Del Moro A. \& Kilias A. 1993. Rb-Sr geochronology and geochemical aspects ofe the Eastern Varnountas plutonite (NW Macedonia, Greece). N. Jb. Miner. Abh. 165, 3, 297-315.

Leake, B. E.,Wooley A. R., Arps C. E. S., Birch W. D. Gilbert, M. C. Grice J. D., Hawthorne F. C., Kato A., Kisch H. J., Krivovichev V. G., Lintholit, K., Laird J., Mandarino J. A., Maresh W. V., Nickel, E. H., Rock N. M. S., Schumacher J. C., Smith D. C., Stephenson N, C., N., Ungaretti L., Whittakr E. J. W. \& Youzhi G. 1997. Nomenclature of Amphiboles: Report of the Subcommittee on Amphiboles of the International Mineralogical Association, Commission on New Minerals and Mineral Names. Can. Mineral., 35, 219-246.

Luhr, J. F. \& Carmichael, I. S. E. 1980. The Colima Volcanic Complex, Mexico. I. Post-caldera Andesites from Volcan-Colima. Contrib. Mineral. Petrol., 71, 343-372.

Luhr J. F., Carmichael I. S. E. \& Varecamo J. C. 1984. The 1982 eruption of El Chichon volcano, Chiapas, Mexico: mineralogy and petrology of the anhydrite bearing pumices. J. Volcanol. Geotherm. Res., 23, 69108.

Mountrakis D. 1986. The Pelagonian zone in Greece: A polyphase-deformed fragment of the Cimmerian continent and its role in the geotectonic evolution of the eastern Mediterranean. Journal of Geology, 94, 335-347.

Mahood, G. and Hildreth, W. 1983. Large partition coefficients for trace elements in high- silica rhyolites. Geochim. Cosmochim. Acta, 47, 11-30.

Mahood G. A. \& Stimac J. A. 1990. Trace-element partitioning in pantellerites and trachytes. Geochim. Cosm. Acta, 54, 2257-2276.

Massone J. H \& Schreyer W. 1987. Phengite geobarometry based on the limiting assemblage K-feldspar, phlogopite and quartz. Contrib. Mineral. Petrol., 96, 212-224.

Most T., Frisch W., Dunkl I., Kadosa B., Boev B., Avgerinas A. \& Kilias A. 2001. Geochronological and structural investigations of the Northern Pelagonian crystalline zone. Constraints from K/Ar and zircon and apatite fission track dating. Bull. Soc. Geol. Greece, 64/1, 91-95.

Mposkos E. \& Perraki M. 2001. High pressure Alpine metamorphism of the Pelagonian allochthon in the Kastania area (Southern Vermion), Greece. Bull. Soc. Geol. Greece, 64/3, 939-947.

Nagasawa H. \& Schnetzler C. C. 1971. Partitioning of rare earth, alkali and alkaline earth elements between phenocrysts and acidic igneous magma. Geochim. Cosm. Acta, 35, 953-968.

Nash, W. P. \& Crecraft, H. R. 1985. Partition coefficients for trace elements in silicic magmas. Geochim. Cosmochim. Acta, 49, 2309-2322.

Pearce J.A., Harris N.B.W. \& Tindle A.C. 1996. Trace element discrimination diagrams for the tectonic interpretation of granitic rocks. J. Petrol., 25, 4, 956-983.

Rollinson, H. 1993. Using geochemical data: evaluation, presentation interpretation. Longman Group UK Ltd., 352pp.

Streckeisen A. \& Le Maitre R.W. 1979. A chemical approximation to the madal QAPF classification of the igneous rovks. N. Jb. Min. Abh., 136, 169-206.

Soldatos, T., Poli, G., Christofides, G, Eleftheriadis, Koroneos, A. \& Tommasini, S. 1998. Petrology and evolution of transitional alkaline-subalkaline granitoids from Vrondou (NE Greece): evidence for fractional crystallization and magma mixing. In: "Tertiary magmatism of the Rhodopian region", Christofides, G., Marchev, P. \& Serri, G. (Eds), Spec. vol., Acta Vulcanologica, 10, 2, 319-330. 


\title{
GEOCHEMISTRY, GENESIS AND STRUCTURE OF HERCYNIAN GRANITOID ROCKS OF THE PELAGONIAN ZONE, FROM THE VORAS MT. (MACEDONIA, NORTHERN GREECE)
}

\author{
Avgerinas A. ${ }^{1}$, Kilias A. ${ }^{1}$, Koroneos $A .{ }^{2}$ and Mountrakis $D .{ }^{1}$ \\ ${ }^{1}$ Department of Geology, asterix@geo.auth.gr, kilias@geo.auth.gr \\ ${ }^{2}$ Department of Mineralogy-Petrology-Economic Geology, koroneos@geo.auth.gr
}

School of Geology, Aristotle University of Thessaloniki, 5462

\begin{abstract}
The Ercynian plutonic rocks intruding the Paleozoic basement of the Pelagonian zone in the area of Kaimaktsalan (western Voras Mt.) are examined. They are composed of: hornblende-biotite granodiorites to granites ( $\mathrm{Hb}) \mathrm{BtGrd}$, biotite granites (BtGr), monzonitic to monzodioritic mafic enclaves (MME) and leucogranites ( $\mathrm{LGr}$ ). The ( $\mathrm{Hb}) \mathrm{BtGrd}$ and $\mathrm{BtGr}$ are randomly distributed and exhibit transitional contacts, while both are intruded by the LGr. In the ( $\mathrm{Hb}) \mathrm{BtGrd}$ two types of biotites were found; one similar to MME biotites and another similar to BtGr. The amphiboles are magnesiohornblende and edenite. The $\mathrm{Nb}$ vs. $\mathrm{Y}$ and $\mathrm{Rb}$ vs. $\mathrm{Y}+\mathrm{Nb}$ plots of the rock-samples indicate the strong relationship of both $(\mathrm{Hb}) \mathrm{BtGrd}$ and $\mathrm{BtGr}$ with a volcanic arc (VAG) geotectonic setting, while the $\mathrm{LGr}$ are plotted in the syn-collision (syn-COLG) field. Both the (Hb)BtGrd and BtGr are plotted in the field of the pre- or post-collisional granites, whereas the $\mathrm{LGr}$ reveal the characteristics of the syn-collisional anatectic granites (R1-R2 diagram). The majority of the major elements show wellcorrelated trends with differentiation starting from the more basic MME up to the $\mathrm{LGr}$ or the $\mathrm{BtGr}$ while, the behaviour of trace elements is characterized by significant changes between MME and (Hb)BtGrd+BtGr. The Kaimaktsalan granitoids show calc-alkaline affinity. The $\mathrm{Rb} / \mathrm{Sr} v \mathrm{vs} .1 / \mathrm{Sr}$ and $\mathrm{Rb} / \mathrm{N}$ vs. $1 / \mathrm{V}$ display straight line trends for $(\mathrm{Hb}) \mathrm{BtGrd}+\mathrm{BtGr}$ indicative of mixing processes.

Based on the field observations, mineralogical composition and geochemistry of the rock types, a two-stage process for the formation of MME, $(\mathrm{Hb}) \mathrm{BtGrd}$ and $\mathrm{BtGr}$ is suggested. In the first stage, a basic magma of mantle origin, and composition similar to the more basic MME underwent fractional crystallization, while it was mixed with an acid magma of crustal origin and composition similar to the most acid $\mathrm{BtGr}$. This mixing-fractional crystallization (MFC) process has low $r$, due to the difference in composition and viscosity of the two magmas. This process resulted in the formation of the most basic $(\mathrm{Hb})$ BtGrd. In the second stage, the resulted magma was fractionated and mixed with the fore mentioned crustal magma, but with higher ( $r$ ) giving the $(\mathrm{Hb}) \mathrm{BtGrd}$ and $\mathrm{BtGr}$. Sub-parallel and crosscutting trends between the $\mathrm{LGr}$ and BtGr suggest that the former do not represent an evolved magma of the latter, but a separated intrusion.
\end{abstract}

Four main deformational events $\left(D_{1}, D_{2}, D_{3}\right.$ and $\left.D_{4}\right)$ of Alpine age and plastic to semi-plastic conditions affected the crystalline basement of the Pelagonian zone in the broader area of the Voras Mt. as well as the Ercynian plutonic rocks. The $\mathrm{D}_{1}$-event is related to a Late Jurassic-Lower Cretaceous $\mathrm{S}_{1}$-schistosity associated with $\mathrm{M}_{1}$-paragenesis of $\mathrm{Qtz}+\mathrm{Kf}+\mathrm{Ab}+\mathrm{Phe}+\mathrm{Bt}+\mathrm{Hb}+$ $\mathrm{Ep} / \mathrm{Czo} \pm$ Tit \pm Garn. During the Middle Cretaceous the $\mathrm{S}_{1}$-schistosity was overprinted by an $\mathrm{S}_{2}$-axial plane schistosity of tight, recumbent to inclined $B_{2}$-folds $\left(D_{2}\right.$-event). The $S_{2}$-schistosity has NW-SE strike and dips either towards the SW or NE. It is characterized by the syn-kinematic growth of $\mathrm{Wm}+\mathrm{Chl}+\mathrm{Ab}+\mathrm{Act}+\mathrm{Qtz}+\mathrm{Ep}\left(\mathrm{M}_{2}\right.$-paragenesis) that forms an $\mathrm{L}_{2}$-stretching lineation striking $\mathrm{NW}-\mathrm{SE}$ closely related with a transport direction towards the SE. The M1-paragenesis along the $S_{1-}$ schistosity indicates that the $\mathrm{D}_{1}$ event took place in the upper greenschist-lower amphibolitic metamorphic facies $\left(\sim 450-550^{\circ} \mathrm{C}\right)$. Also, the composition of the syn- $S_{1}$ phengite (max Si: 6,8$)$ shows that the granites and orthogneisses of the crystalline basement of Kaimaktsalan have been metamorphosed in relatively high-pressure conditions $(\sim 8 \mathrm{~kb})$. The replacement of $\mathrm{Bt}$ with $\mathrm{Chl}$ and $\mathrm{Hb}$ with Act and $\mathrm{Chl}$ indicates that the $\mathrm{M}_{2}$-paragenesis took place below the $\sim 450^{\circ} \mathrm{C}$. The $\mathrm{D}_{2}$-event was succeeded by a younger $D_{3}$-event that took place in semi-plastic conditions in Late Cretaceous. The $\mathrm{D}_{3}$-event is characterized by NNW-SSE to NE-SW extensional $\mathrm{Sb}_{3}$-shear zones and a stretching lineation associated with a top to the SE sense-of-shear. During the Tertiary and in more cold conditions, NW-SE striking $\mathrm{Sb}_{4}$-shear zones with ultra-cataclastites and sense-of-shear mainly top to the $\mathrm{SW}$ were formed due to the $\mathrm{D}_{4}$-event affecting all the previous structures. 